Article

\title{
EntangleNet: Theoretical Reestablishment of Entanglement in Quantum Networks ${ }^{\dagger}$
}

\author{
Mihai-Zicu Mina ${ }^{\ddagger}$ and Pantelimon George Popescu ${ }^{\ddagger}, *$ \\ Computer Science and Engineering Department, Faculty of Automatic Control and Computers, \\ University Politehnica of Bucharest, 060042 Bucharest, Romania; mihai_zicu.mina@aut.pub.ro \\ * Correspondence: pgpopescu@yahoo.com \\ + To Octavian Stănășilă on his 80th birthday. \\ $\ddagger$ These authors contributed equally to this work.
}

Received: 6 September 2018; Accepted: 14 October 2018; Published: 16 October 2018

\begin{abstract}
In the practical context of quantum networks, the most reliable method of transmitting quantum information is via teleportation because quantum states are highly sensitive. However, teleportation consumes a shared maximally entangled state. Two parties Alice and Bob located at separate nodes that wish to reestablish their shared entanglement will not send entangled qubits directly to achieve this goal, but rather employ a more efficient mechanism that ensures minimal time resources. In this paper, we present a quantum routing scheme that exploits entanglement swapping to reestablish consumed entanglement. It improves and generalizes previous work on the subject and reduces the entanglement distribution time by a factor of $4 k$ in an arbitrary scale quantum network, where $N=4 k-1$ is a required number of quantum nodes located between source and destination. In addition, $k$ is the greatest positive integer considered by Alice or Bob, such that afterwards they choose $N$ quantum switches.
\end{abstract}

Keywords: quantum teleportation; quantum network; quantum routing; entanglement swapping

\section{Introduction}

Quantum teleportation is a communication protocol introduced by Bennett et al. [1] and represents a major result in the field of quantum information. It allows perfect transmission of an unknown qubit between two spatially separated parties (whom we shall call Alice and Bob), provided that classical information is exchanged and a previously shared maximally entangled pair of particles exists between them [1-3]. Quantum entanglement is one of the most peculiar aspects exhibited by nature and an essential resource for teleportation. Shared maximal entanglement is required to achieve faithful teleportation; however, an inherent limitation is that this highly valuable resource is lost afterwards. In such context, shared entanglement as an informational resource is expressed in a quantitative manner as one entanglement bit or 1 ebit [2,4]. The teleportation scheme is part of a paradigm known as local operations and classical communication (LOCC), with additional shared entanglement allowed. This means that Alice and Bob share an entangled pair of particles, can manipulate their own qubits at will (LO) and can only exchange classical bits of information (CC). An important drawback of the protocol is closely related to properties of entanglement under LOCC; specifically, one shared ebit is necessary for faithful teleportation and the amount of shared entanglement cannot be increased by local operations and classical communication [2,4-8]. A consequence of these constraints is the consumption of one shared ebit when teleporting one qubit, thus entanglement between Alice and Bob is lost.

Alice and Bob are thought to be part of an extended quantum network infrastructure, with quantum channels providing communication links between adjacent nodes. Physical transmission of particles 
through these inherently noisy channels will definitely alter the encoded quantum information they wish to exchange. Teleportation proves to solve this problem by accurately transmitting a qubit from one place to another, regardless of distance and without any physical transport. However, it requires a maximally entangled pair of particles to be shared between source and destination; therefore, a crucial aspect of a future large scale quantum network is the task of reliably distributing entanglement to any two nodes in need of exchanging quantum information via teleportation.

We have chosen to devise a theoretical entanglement distribution protocol based on quantum routing that manages to resupply any two nodes Alice and Bob in a quantum network with shared entanglement. Our result is a generalization of [9] that reduces the entanglement distribution time by a factor of $4 k$ relative to direct transmission of entangled qubits, considering a number of $4 k-1$ specifically chosen quantum nodes between source and destination, as detailed in Section 2.2.

This paper is organized according to the following structure. In Section 2, we explore the process of entanglement distribution in quantum networks and discuss already established results. Then, we present our idea that improves and generalizes previous results, along with a resource analysis regarding the time needed to supply the end nodes with a maximally entangled pair of qubits. In Section 3, we provide an algorithmic description of the protocol and in Section 4 we conclude with final remarks and discuss future work.

\section{The Idea of Restoring Entanglement}

\subsection{General Context}

In a general case of entanglement distribution, Alice and Bob usually have quantum channels connecting them to Charlie, another node in the network. We refer to Charlie as a third party, who might actually represent a laboratory that generates maximally entangled pairs and then sends each particle to Alice and Bob, thus having successfully provided them with shared entanglement. Nevertheless, problems arise when considering practical situations of entanglement distribution. Although one ebit constitutes a perfect, noiseless transmission channel for Alice and Bob [10], the entangled pair is still distributed via noisy quantum channels, hence its purity is altered and our parties will eventually share a non-maximally entangled state (less than $1 \mathrm{ebit}$ ). By executing the teleportation protocol with such a resource, perfect reconstruction of the original qubit cannot be accomplished.

A major result that overcomes these limitations is the process of entanglement purification [2,10], which is possible via local operations. The purpose of purification protocols is to concentrate the entanglement of a certain number of entangled pairs to obtain a higher amount of entanglement for a smaller number of pairs. Given sufficient partially-entangled pairs shared by Alice and Bob, under LOCC, they can obtain a pair with entanglement close to 1 ebit in order to teleport a qubit with satisfactory fidelity. Entanglement concentration using nonlinear optics is presented in [11].

Even though entanglement can be concentrated, this process is successful under a minimum fidelity condition for the initial pairs, which cannot be met beyond certain distances [10,12]. It is reasonable to assume that distances between such arbitrary nodes as those represented by Alice and Bob are significant enough to make this process rather infeasible. This led to the emergence of quantum repeaters [12], devices that use entanglement swapping [13-15] and purification in order to propagate entanglement in shorter segments (node to node), until source and destination are linked by an almost maximally entangled pair. Thus, quantum repeaters are key elements in the framework of a quantum network and they have been considered in various entanglement distribution schemes. The phenomenon of entanglement swapping for continuous variables is discussed in [16]. A quantum key distribution scheme involving entanglement swapping is proposed in [17], while a quantum conferencing scheme for secure communication between several users is developed in [18].

An entanglement distribution scheme was recently proposed as an optimal routing protocol that ensures the greatest entanglement distribution rate between two arbitrary nodes in a quantum network [19]. It operates on a graph model of the network, while taking into account physical 
challenges such as the stochastic nature of entanglement generation, imperfect Bell measurement and short coherence time $T^{\mathrm{ch}}$ of memories in quantum repeaters. Given multiple possible routes between two nodes, the protocol finds the optimal one according to a route metric. The optimality of the path provides the highest average number of successfully established entangled pairs between two nodes in unit time, considering the coherence time interval $T^{\mathrm{ch}}$. Through numerical simulations, the underlying algorithm of the protocol was proven to outperform classical graph algorithms (Dijkstra, Bellman-Ford) used in conventional routing.

Two other previously proposed routing schemes are referred to as quantum relay and EPR-pair bridging [20] (maximally entangled pairs of particles are also called EPR-pairs). Considering Alice and Bob do not share entangled pairs with each other, but rather each of them does with another node Charlie, then a quantum relay method achieves teleportation of a qubit from Alice to Bob in two steps: Alice teleports first to Charlie, followed by him teleporting to Bob. By extension, an arbitrary number of intermediate nodes can exist between Alice and Bob, with entangled pairs set up between adjacent stations. This hop-by-hop "forwarding" mechanism exhibits an obvious lack of security and privacy [20], as the initial quantum information passes through all the nodes before finally reaching its intended destination. EPR-pair bridging addresses this problem by implementing a swapping scheme that provides Alice and Bob with a shared entangled pair. A Bell measurement performed by Charlie on his qubits and a subsequent transmission of classical information will establish entanglement as a particular Bell state between qubits held by Alice and Bob. Using this method based on entanglement swapping, direct teleportation is realized between source and destination. A quantum routing protocol was considered as a combination of quantum relay and EPR-pair bridging mechanisms, without necessary time synchronization between nodes, as a faster completion of operations at source or at the intermediate nodes would degenerate into the former or the latter scheme, respectively [20].

Entanglement swapping is a phenomenon of paramount importance for the entanglement distribution process between two distant nodes. In a most elementary scenario, we consider that there is a node $O$ halfway between Alice $(A)$ and Bob $(B)$ that can generate entangled pairs of particles and then distribute them accordingly. This distribution scheme can be improved, as proposed in [9]. It was demonstrated that a significant speedup is exhibited when further nodes are introduced on the routes $A-O$ and $O-B$. Considering Alice and Bob are separated by a distance $L$ and assuming entangled qubits travel at a speed $v$, the time required for our parties to receive their qubits generated at $O$ is no less than $t_{1}=L / 2 v$. By placing distribution nodes $C$ and $D$ halfway on paths $A-O$ and $O-B$, they generate entangled pairs and simultaneously send a qubit to $O$ and the other one to $A$ and $B$, respectively. The time generated qubits require to arrive at their destination nodes is reduced to $L / 4 v$. Following a Bell measurement performed at $O$ that takes $t_{m}$ to complete, Alice and Bob finally share entanglement. Without accounting for the time necessary for classical information associated with the measurement to be received, then $A$ and $B$ are supplied after an interval of $t_{2}=L / 4 v+t_{m}$, which is less than $t_{1}$, provided $t_{m}<L / 4 v$.

Having analyzed the framework of the problem and several existing protocols, we now continue with presenting our approach to how entanglement can be restored between Alice and Bob in a practical quantum network scenario, improving and generalizing previous results.

\subsection{Our Proposal}

Given the already existing network infrastructure and taking into account financial costs, a future large scale quantum network would most likely be mapped onto it. Therefore, optical fiber links will represent quantum channels and most nodes will likely use quantum equipment to achieve various tasks. Our solution is formulated in such a context and has at the very basis the idea described in [9], which we improve and generalize. We now define our general model of the network and the elements that are part of our scheme.

We consider a hybrid network (having both classical and quantum nodes) with a graph-like topology. Each component node is identified by an address (as an IP address), it is linked by a quantum 
channel to at least one other node and has a particular status, i.e., it is tasked with accomplishing a certain operation. An important class of nodes we consider is represented by quantum switches (QS), devices that are able to execute quantum routing protocols, generate entangled pairs of particles and perform various types of measurements on qubits. Our parties Alice and Bob are two nodes separated by an arbitrary distance that frequently exchange quantum information via teleportation. Thus, shared entanglement is a key resource they require to achieve their task. After each completed teleportation, their entangled pair is destroyed and another teleportation is not possible. Considering arbitrary distances between end nodes, they cannot afford to locally create entangled pairs of particles and send half of each to their partner. The idea we investigate is how they can be efficiently supplied with another entangled resource. Before discussing it, we present the assumptions based on which the scheme operates.

\section{Assumptions:}

- $\quad$ entanglement generation, Bell measurement (BSM) and other local quantum operations are performed perfectly in a negligible amount of time;

- transmission of entangled qubits between adjacent nodes is realized with high fidelity and does not require purification mechanisms.

These quantum operations are treated as theoretical processes that are abstract. We are not concerned with their physical nature; otherwise, we would not be able to model them as perfect and instantaneous in our scheme. Travel time between nodes can be neglected as well; however, that is not the most important aspect for our analysis. We discuss the time efficiency of the protocol in Section 2.2.2 by considering through how many hops entangled particles must pass until shared entanglement is achieved between the two parties.

We consider that Alice and Bob initially share several entangled pairs of particles described, for example, by Bell state $\left|\beta_{00}\right\rangle=(|00\rangle+|11\rangle) / \sqrt{2}$. After the last teleportation is performed, Alice and Bob acknowledge their resupply need and synchronize with each other in order to send an appropriate classical message to each other at the same time. We label this special message msg_NE_XY, where "NE" stands for "Need entanglement", " $X$ " is the sender and " $Y$ " is the receiver. These two messages may reach their destination by different routes, as depicted in Figure 1. We denote the route taken by each message by the set of nodes it passes through:

- $\quad$ Alice to Bob: $\left\{S_{A_{i}} \mid i=1: m\right\}$

$$
A \stackrel{\text { msg_NE_AB }}{\longrightarrow} S_{A_{1}} \underset{\rightarrow}{\rightarrow} \ldots \stackrel{\rightarrow}{\rightarrow} S_{A_{m}} \stackrel{\text { msg_NE_AB }}{\longrightarrow} B,
$$

- $\quad$ Bob to Alice: $\left\{S_{B_{j}} \mid j=1: n\right\}$

$$
B \stackrel{\mathrm{msg} \_\mathrm{NE}_{-} \mathrm{BA}}{\longrightarrow} S_{B_{1}} \stackrel{\cdots}{\rightarrow} \ldots \stackrel{\cdots}{\rightarrow} S_{B_{n}} \stackrel{\text { msg_NE_BA }}{\longrightarrow} A .
$$

These sets of nodes may coincide; however, this particular circumstance will rarely occur when considering that a continuous level of network activity would make some routes congested, therefore requiring a different route to be found. 


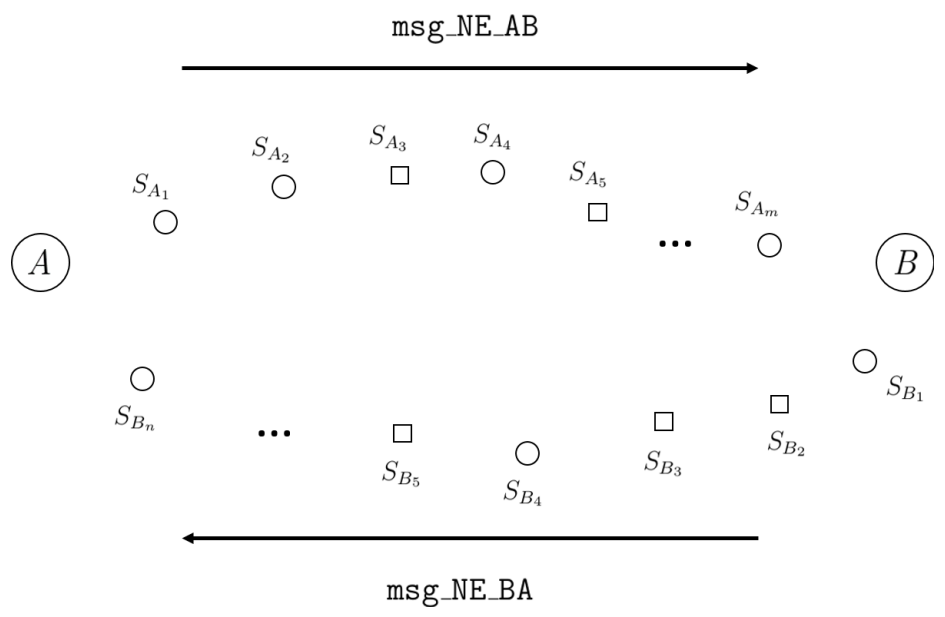

Figure 1. Two possible routes connecting Alice $(A)$ and Bob $(B)$. Circular and rectangular nodes represent quantum and classical switches, respectively.

Among each set of nodes, there is a subset consisting entirely of quantum switches.

$$
\begin{gathered}
\exists\left\{a_{1}, a_{2}, \ldots, a_{p}\right\} \subseteq\left\{A_{1}, A_{2}, \ldots, A_{m}\right\}, \text { such that } \\
\mathcal{S}_{a}=\left\{S_{a_{i}} \mid i=1: p\right\} \text { are QS, } p \leq m, \\
\exists\left\{b_{1}, b_{2}, \ldots, b_{q}\right\} \subseteq\left\{B_{1}, B_{2}, \ldots, B_{n}\right\}, \text { such that } \\
\mathcal{S}_{b}=\left\{S_{b_{j}} \mid j=1: q\right\} \text { are QS, } q \leq n .
\end{gathered}
$$

Each quantum switch is able to interpret the special message msg_NE_XY and subsequently make itself visible to sender $X$ by confirming its status as a quantum node through a classical message sent back to source, msg_ACK_X:

$$
\begin{aligned}
& S_{a_{i}} \stackrel{\text { msg_ACK_A }}{\longrightarrow} A, \\
& S_{b_{j}} \stackrel{\text { msg_ACK_B }}{\longrightarrow} B .
\end{aligned}
$$

Upon receiving confirmation from each quantum switch, Alice and Bob have their own list of IP addresses that describes a route between them consisting exclusively of quantum nodes:

- $\quad$ Route from Alice to Bob (known by Alice):

$A \rightarrow S_{a_{1}} \rightarrow S_{a_{2}} \rightarrow \ldots \rightarrow S_{a_{p}} \rightarrow B$,

- Route from Bob to Alice (known by Bob):

$B \rightarrow S_{b_{1}} \rightarrow S_{b_{2}} \rightarrow \ldots \rightarrow S_{b_{q}} \rightarrow A$.

To begin the distribution process, Alice and Bob each send an encoded classical message, as seen in Table 1, to every node $S_{a_{i}}$ and $S_{b_{j}}$, respectively and also to their partner in order to instruct them on how to act further. This classical information is labeled msg_ACT_A and msg_ACT_B to suggest who it is sent from, Alice or Bob:

$$
\begin{aligned}
& A \stackrel{\text { msg_ACT_A }}{\longrightarrow}\left\{S_{a_{i}}, B\right\}, \\
& B \stackrel{\text { msg_ACT_B }}{\longrightarrow}\left\{S_{b_{j}}, A\right\} .
\end{aligned}
$$


Table 1. Classical instruction messages sent by Alice to her known quantum switches and to Bob.

\begin{tabular}{cl}
\hline Encoding & Significance \\
\hline msg_ACT_A =0 & Do nothing, i.e., route qubit to next node. \\
\hline msg_ACT_A =1 & Generate entangled pair and send one particle to adjacent node. \\
\hline msg_ACT_A = 2 & Generate entangled pair and send each particle to the adjacent nodes. \\
\hline msg_ACT_A = 3 & $\begin{array}{l}\text { Perform Bell measurement on qubits and communicate classical information to } \\
\text { adjacent node. }\end{array}$ \\
\hline msg_ACT_A = 4 & $\begin{array}{l}\text { Generate entangled pair and distribute one particle to adjacent node; perform Bell } \\
\text { measurement on the other particle and the previously received one; communicate } \\
\text { classical outcome to the same node the particle was sent to. }\end{array}$ \\
\hline
\end{tabular}

Our designed scheme operates by isolating the number of active quantum switches (AQS) on a route between source and destination to $N=4 k-1$, for $k \geq 1$. The protocol is symmetric; therefore, we will discuss here the case regarding Alice's perspective.

Considering that $N \leq p$, we may label the selected subset of AQS as $\tilde{\mathcal{S}}_{a} \subseteq \mathcal{S}_{a}$, with $\left|\tilde{\mathcal{S}}_{a}\right|=N$. Quantum switches that are not part of this subset will be referred to as being inactive (IQS). An important aspect must be clarified here. IQS are designated by Alice or Bob in order to restrict the number of quantum switches that actively participate during the protocol to $N=4 k-1 \leq p$. This is how our scheme is devised and it operates on this symmetry argument given by the number $N$ of AQS. There can be at most three IQS and they will be sent an instruction message that makes them operate as classical switches (CS).

For now, we consider that $\tilde{\mathcal{S}}_{a}=\mathcal{S}_{a}$ for brevity in notation (the case when $\tilde{\mathcal{S}}_{a} \subset \mathcal{S}_{a}$ means that $\mathcal{S}_{a}$ differs from $\tilde{\mathcal{S}}_{a}$ by three maximum IQS, which can be considered as classical ones operating just for qubit routing).

According to the choice of $N$, there will be an odd number of such AQS. Following the labeling convention in Equations (1) and (2) and considering the common notation (see [9]), we will refer to the node situated halfway in the set $\mathcal{S}_{a}$ as $O_{A} \equiv S_{a_{2 k}}$. There are $\left\lfloor\frac{N}{2}\right\rfloor=2 k-1$ quantum switches on each of the segments $A-O_{A}$ and $O_{A}-B$. Considering $A \equiv S_{a_{0}}$ and $B \equiv S_{a_{4 k}}$, halfway on the list of quantum switches found on paths $A-O_{A}$ and $O_{A}-B$ are nodes $S_{a_{k}} \equiv A^{\prime}$ and $S_{a_{3 k}} \equiv B^{\prime}$, respectively. Taking into account both paths relative to the position of $O_{A}$, the nodes will act in the following manner:

1. each node from $\mathcal{S}_{a} \backslash\left\{A^{\prime}, B^{\prime}\right\}$ generates a pair of particles $\left(e, e^{\prime}\right)$ in Bell state $\left|\beta_{00}\right\rangle$; Alice and Bob may agree on any Bell state $\left|\beta_{i j}\right\rangle, i j \in\{0,1\}^{2}$ to be shared after the completion of the protocol. To accomplish this task, they would need to exchange classical information encoding the identity of the Bell state (for example, $i j$ ), followed by Alice's instructions sent to every quantum switch she discovered to prepare the appropriate state. The distribution process will then continue as before, making sure that after each BSM the necessary classical information is transmitted between nodes to reconstruct $\left|\beta_{i j}\right\rangle$ each time, so that Alice and Bob share this state in the end.

2. particle $e$ is kept and $e^{\prime}$ is sent to an adjacent node, according to the following rules:

- $\quad$ Path $A-O_{A}$ :

- $\quad$ A towards $A^{\prime}$

$$
\begin{aligned}
& A \stackrel{e_{0}^{\prime}}{\longrightarrow} S_{a_{1}}, \\
& S_{a_{i}} \stackrel{e_{i}^{\prime}}{\longrightarrow} S_{a_{i+1}}, \quad 1 \leq i \leq k-1,
\end{aligned}
$$


- $O_{A}$ towards $A^{\prime}$

$$
\begin{aligned}
& S_{a_{2 k-1}} \stackrel{e_{2 k}^{\prime}}{\longleftarrow} O_{A}, \\
& S_{a_{i-1}} \stackrel{e_{i}^{\prime}}{\longleftarrow} S_{a_{i}}, \quad k+1 \leq i \leq 2 k-1,
\end{aligned}
$$

- $\quad \operatorname{Path} O_{A}-B$ :

- $O_{A}$ towards $B^{\prime}$

$$
\begin{aligned}
& O_{A} \stackrel{e_{2 k}^{\prime}}{\longrightarrow} S_{a_{2 k+1}}, \\
& S_{a_{i}} \stackrel{e_{i}^{\prime}}{\longrightarrow} S_{a_{i+1}}, \quad 3 k-1 \leq i \leq 2 k+1,
\end{aligned}
$$

- $\quad$ B towards $B^{\prime}$

$$
\begin{aligned}
& S_{a_{4 k-1}} \stackrel{e_{4 k}^{\prime}}{\longleftarrow} B, \\
& S_{a_{i-1}} \stackrel{e_{i}^{\prime}}{\longleftarrow} S_{a_{i}}, \quad 3 k+1 \leq i \leq 4 k-1 .
\end{aligned}
$$

We make an important remark here. According to the particle flow previously represented, Alice sends one particle of her EPR pair to her nearest node, which is a quantum one. Halfway node $O_{A}$ and Bob's nearest node $S_{N}$ are also quantum nodes. This choice makes the notation consistent, although that node might as well be a classical switch, in which case it would just forward the qubit until it reaches a quantum switch. IQS and CS are not explicitly present in these representations, but they assist in the dynamics of the scheme.

A distribution of entanglement performed in a manner that does not imply swapping is depicted in Figure 2. Alice initially prepares her entangled pair of qubits and then sends one particle to her nearest node, which in turn forwards it to its neighbor and so on. There are $N+1=4 k$ hops required during this process for Bob to receive the particle. Assuming each hop is realized in one unit of time, then Alice and Bob will share entanglement after $t_{0} \equiv 4 k$ units of time.

Based on these distribution rules, there are four path segments from $A$ to $B$ as the following sets:

- $R_{1}=\{1, \ldots, k-1\}$,

- $R_{2}=\{k+1, \ldots, 2 k-1\}$,

- $R_{3}=\{2 k+1, \ldots, 3 k-1\}$,

- $R_{4}=\{3 k+1, \ldots, 4 k-1\}$.

Then, as expressed in (3)-(6), $R_{F} \equiv R_{1} \cup R_{3}$ and $R_{B} \equiv R_{2} \cup R_{4}$ contain indices that identify active quantum switches on forward and backward distribution routes, respectively, as shown in Figure 3.

Considering that a classical switch cannot execute quantum-specific operations, it will not require a particular instruction message, since it can only route the received qubit further until it reaches a quantum switch.

The main goal of the protocol is to ensure particles reach nodes $A^{\prime}$ and $B^{\prime}$ via both upstream (from $A$ and $B$ towards $O_{A}$ ) and downstream (from $O_{A}$ towards $A$ and $B$ ) routes. Considering each intermediate quantum switch, i.e., each node from $\mathcal{S}_{a} \backslash\left\{A^{\prime}, O_{A}, B^{\prime}\right\}$ has successfully received the qubit from its nearest node and has itself sent one from its generated pair, then it performs a BSM on the two qubits from different pairs to accomplish entanglement swapping, thus propagating the entanglement towards middle nodes $A^{\prime}$ and $B^{\prime}$. Additional classical information resulting from the measurement is required to be provided to the destination node in order to preserve the original Bell state $\left|\beta_{00}\right\rangle$. This step is completed by applying an appropriate corrective operation $U \in\left\{I, \sigma_{x}, \sigma_{z}, \sigma_{z} \sigma_{x}\right\}$ to the received qubit. These two nodes $A^{\prime}$ and $B^{\prime}$ will be the last ones to possess two qubits received from upstream and downstream. A subsequent BSM performed by both of them on their qubits will then establish entanglement between Alice and Bob, therefore achieving the original task. We remark that a 
total of $N-1=4 k-2$ Bell measurements are performed during the entire process, along with the same number of classical messages sent to assist in adjusting the received state to be the correct one. A visual representation of the the distribution process is shown in Figure 4.

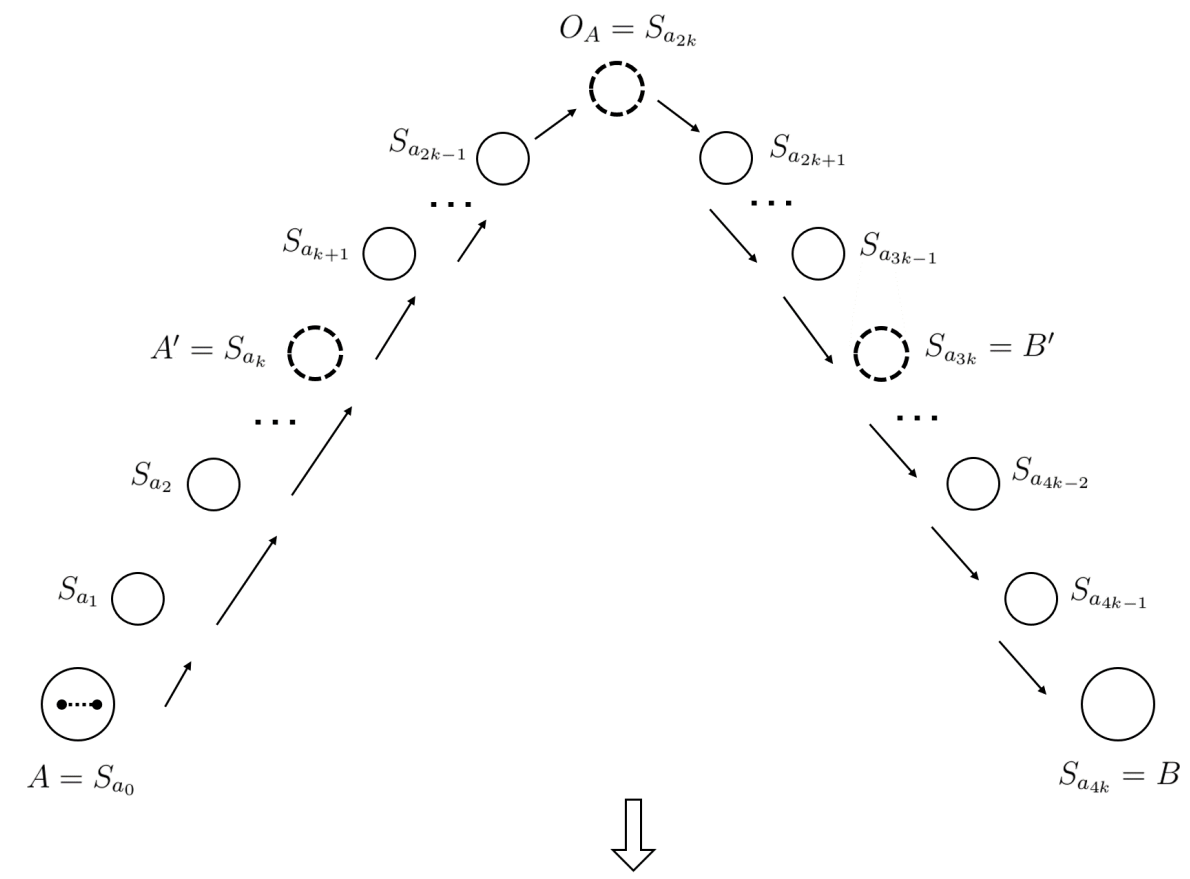

After $4 k$ hops

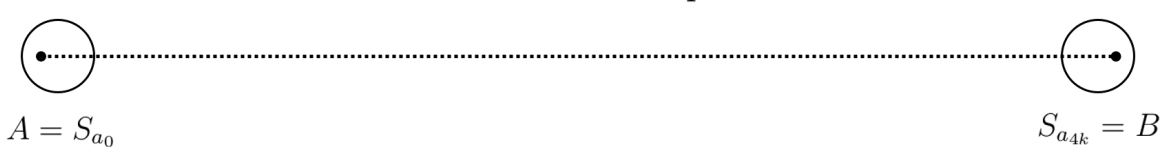

Figure 2. Tree-like illustration of a configuration of $N=4 k-1$ active quantum switches on route from $A$ to $B$ (tilde notation dropped to avoid cluttering). Dotted black lines connect entangled particles, as it is initially seen at Alice. A sequential distribution of entanglement from Alice to Bob implies that one particle of Alice's entangled pair goes through $4 k$ nodes to eventually reach Bob. This process would take $t_{0}=4 k$ units of time.

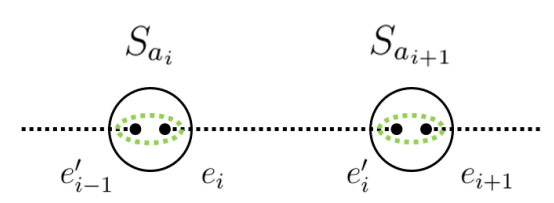

Forward distribution (routes $A-A^{\prime}$ and $O_{A}-B^{\prime}$ )

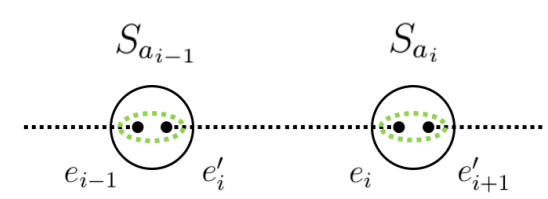

Backward distribution (routes $B-B^{\prime}$ and $O_{A}-A^{\prime}$ )

Figure 3. Creation of entangled pairs at each node from the subset of $N$ active quantum switches. Depending on the position relative to middle stations $A^{\prime}$ and $B^{\prime}$, a qubit is received from downstream or upstream, while one qubit of the generated pair is further sent to another node. Bell measurements (dotted ellipse around particles) will eventually be performed on qubits from different entangled pairs. 


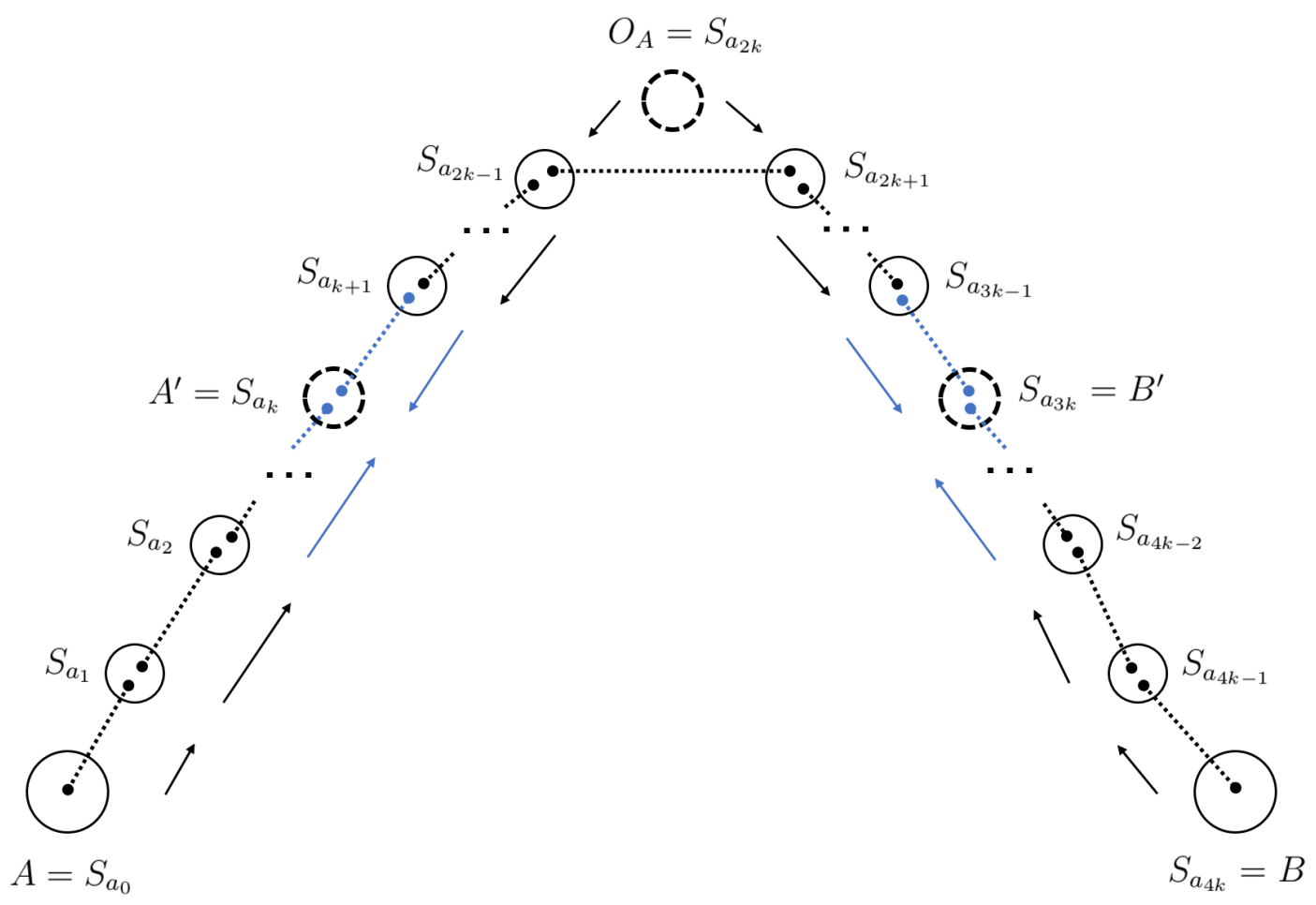

Figure 4. The distribution process of our scheme converges to $A^{\prime}$ and $B^{\prime}$, where the last Bell measurements are performed, as indicated by blue arrows and blue colored entangled qubits that arrive there.

As previously mentioned, the scheme we have presented takes advantage of a configuration of $N=4 k-1$ quantum switches between Alice and Bob. Thus, $p-N$ quantum switches will not participate in the protocol. Since each active quantum switch must receive a qubit, be able to generate an entangled pair and then send one qubit to the next quantum switch, the inactive $p-N$ ones will act as classical switches and only route the qubit further, until another AQS is reached. Under these circumstances, there will be at most three IQS on the route from Alice to Bob:

- $p=4 k-1=N \Longrightarrow$ all QS are AQS,

- $p=4 k \quad \Longrightarrow p-N=1$ IQS,

- $p=4 k+1 \quad \Longrightarrow p-N=2$ IQS,

- $p=4 k+2 \Longrightarrow p-N=3$ IQS,

- $\quad p=4 k+3=4(k+1)-1=4 k^{\prime}-1=N^{\prime}$.

Figure 5 depicts a scenario that involves two IQS between two AQS.

After receiving Alice's request message msg_NE_A, each QS sends an acknowledgment message back to Alice, revealing its address to her. When there is a non-zero difference between the number of QS $(p)$ and the number of AQS $(N)$, Alice makes a decision regarding which switches will participate in the protocol. Those $p-N$ excluded will be sent a classical message msg_ACT_A $=0$ to assist those AQS just by forwarding the qubits received from them. She identifies nodes $A^{\prime}, O_{A}$ and $B^{\prime}$ in order to properly instruct the remaining ones about the distribution rules. Bob $(B)$ will receive an instruction msg_ACT_A $=1$ (the corresponding operation is also performed by Alice), while $O_{A}$ will receive msg_ACT_A $=2$. Nodes $A^{\prime}$ and $B^{\prime}$ will receive from Alice msg_ACT_A $=3$ to denote their BSM operation. The remaining AQS must generate entangled pairs and then perform a BSM, an operation which is communicated as msg_ACT_A $=4$. 
Alice generates her entangled pair and sends one particle to the nearest node; then, she waits for the protocol to execute and finally receives two bits of information from $A^{\prime}$ after its measurement, in order to recover the accurate Bell state. On Bob's segment, $B^{\prime}$ also provides him with this classical information. After these last two measurements, Alice and Bob share an entangled pair in Bell state $\left|\beta_{00}\right\rangle$ to be used in yet another teleportation they wish to perform.

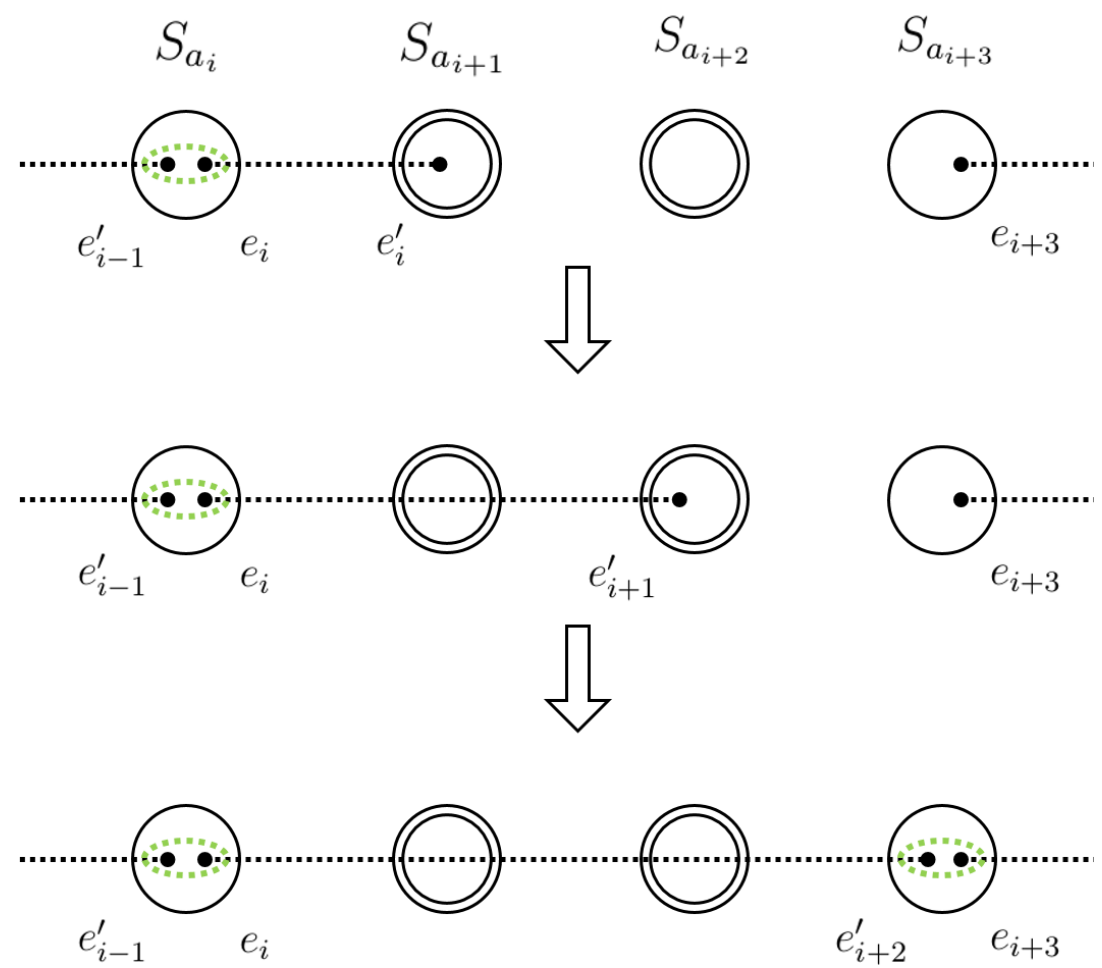

Figure 5. A scenario that involves two inactive quantum switches (concentric circles) between two active ones (circular shapes) on a forward distribution segment. Active quantum switch $S_{a_{i}}$ follows the protocol and sends qubit $e_{i}^{\prime}$ to next node $S_{a_{i+1}}$. The receiver is not part of the chosen subset to create entangled pairs or measure qubits, thus it forwards the particle to $S_{a_{i+2}}$. The particle is again routed to node $S_{a_{i+3}}$, which is active and can therefore perform a Bell measurement on its qubits.

\subsubsection{Exemplification}

A representation of the protocol for the first and second particular cases is depicted in Figures 6 and 7, respectively. It should be noted that CS and IQS are not pictured explicitly; however, their actions contribute to the functionality of the scheme. In Figure 6, we consider $k=1$, so there are three AQS between Alice and Bob. Nodes $A, O_{A}, B$ distribute generated entangled qubits directly to $A^{\prime}$ and $B^{\prime}$, which then perform a BSM and provide Alice and Bob with entanglement. In Figure 7, we consider $k=2$; therefore, seven AQS are present on the path from Alice to Bob. This time the distribution of particles propagates towards $A^{\prime}$ and $B^{\prime}$ by intermediate Bell measurements performed by the other AQS. Finally, $A^{\prime}$ and $B^{\prime}$ are the last ones to perform a BSM, which entangles the qubits kept by Alice and Bob. 


$$
k=1
$$

\section{Preparation}

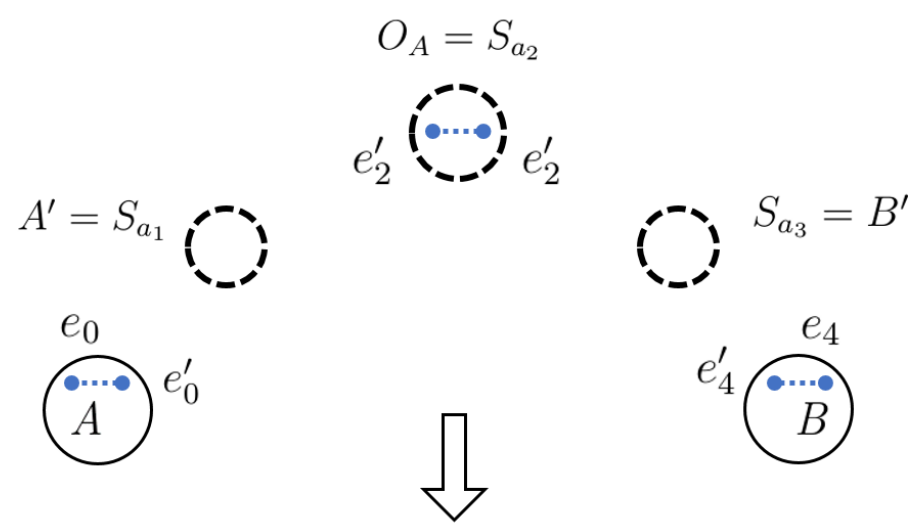

\section{Distribution in 1 unit of time}

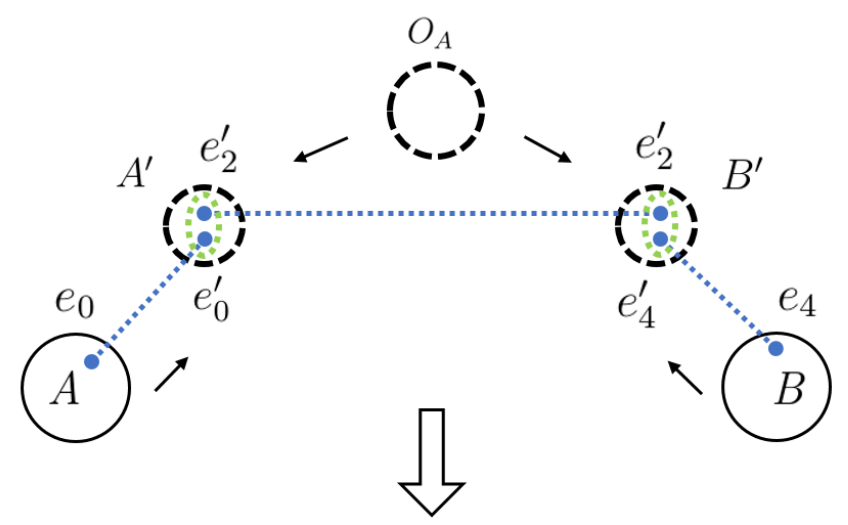

Bell measurement

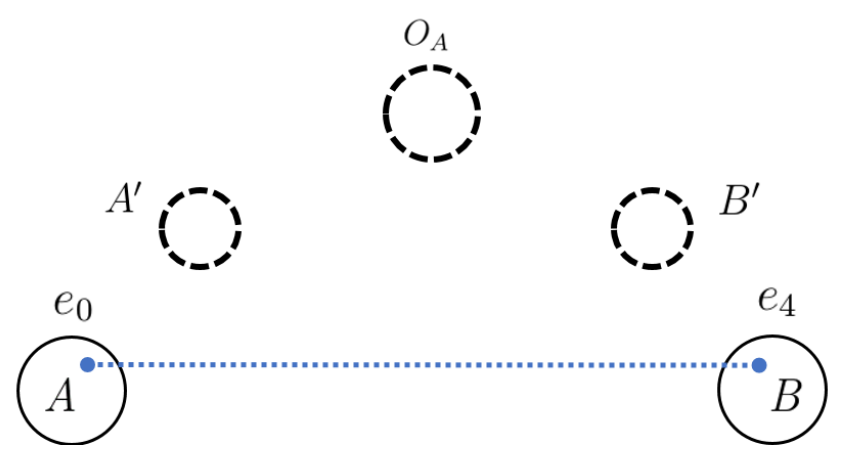

Figure 6. An illustration of the protocol for $k=1$. Nodes $A, O_{A}$ and $B$ locally prepare entangled pairs of qubits. Nodes $A^{\prime}$ and $B^{\prime}$ each receive two qubits (from $A$ and $O_{A}$ and from $O_{A}$ and $B$, respectively). A Bell measurement can then be performed on the qubits from different entangled pairs, regardless of the order, followed by classical communication of outcome (not pictured). For example, $A^{\prime}$ is the first to perform a Bell measurement, followed by $B^{\prime}$. After these steps, entanglement has propagated via swapping towards Alice and Bob, who finally share one ebit as entangled pair $\left(e_{0}, e_{4}\right)$. 


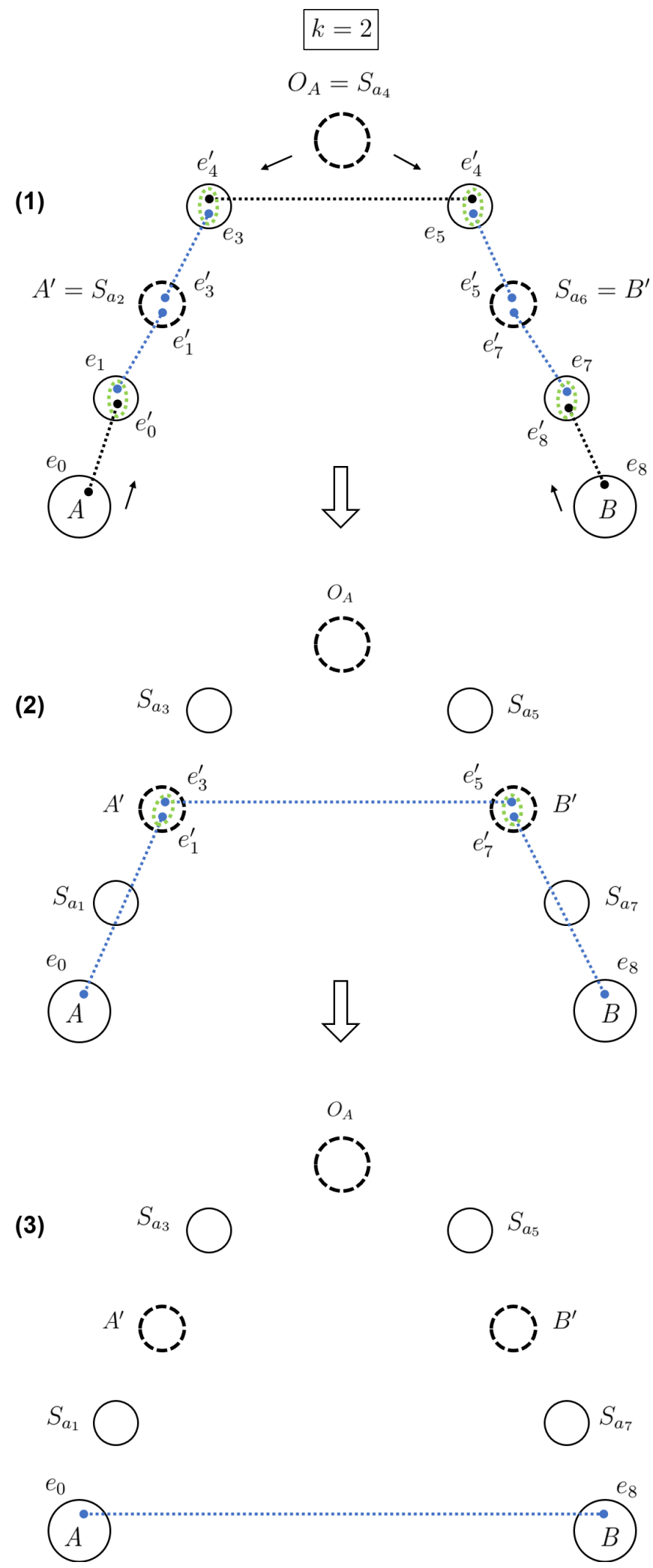

Figure 7. Example for $k=2$. Nodes $A^{\prime}$ and $B^{\prime}$ can no longer be reached directly by $A, B$ or $O_{A}$. Links of entangled pairs are established according to the instructions received by each node. Without needing to be synchronized, Bell measurements are performed at each station on the four segments of the path (1). Middle nodes $A^{\prime}$ and $B^{\prime}$ can execute the same task after receiving the qubits from their adjacent nodes (2), thus providing $A$ and $B$ with entangled pair of particles $e_{0}$ and $e_{8}(3)$. 


\subsubsection{Efficiency}

We continue with emphasizing a feature of our proposal based on a time efficiency analysis. The general scenario involves the existence of these $N=4 k-1$ active quantum switches between Alice and Bob. As depicted in Figure 2, the straightforward approach of Alice preparing an entangled pair and sending the other particle to Bob is done in $4 k$ units of time, an interval we label as $t_{0}$. When the quantum switch $O_{A}$ between $A$ and $B$ is employed in the protocol, it prepares its entangled pair and sends each particle to them. Thus, the time required for each qubit to reach its destination is reduced to $t_{1}=t_{0} / 2$, assuming the intermediate node is located halfway on the route from Alice to Bob and qubits travel at the same speed.

The first particular case of our protocol is $k=1$, for which there are $N=4 k-1=3$ nodes between Alice and Bob that participate in the distribution, i.e., one additional quantum switch found halfway on the segments $A-O_{A}$ and $O_{A}-B$. According to the steps of the scheme, qubits must arrive at $A^{\prime}=S_{a_{1}}$ and $B^{\prime}=S_{a_{3}}$. A BSM is then performed at these stations, followed by $N-1=2$ (two) transmissions of classical information as part of entanglement swapping. Considering this classical information takes a time $t_{c i}$ to reach its destination and the transmissions occur in parallel, we observe that Alice and Bob will have their desired pair in an interval of $t_{N}=t_{3}=t_{0} / 4+t_{c i}$.

As the number of quantum switches $N$ increases, we derive the general relation that describes the time resources needed to supply Alice and Bob with entanglement,

$$
t_{N}=\frac{t_{0}}{N+1}+t_{c i}=\frac{t_{0}}{4 k}+t_{c i}, \quad k \geq 1 .
$$

As $N$ becomes larger, the distance between quantum switches will be short enough to consider $t_{c i}$ negligible. Therefore, the protocol reduces the distribution time by a factor of $N+1=4 k$ relative to direct transmission of qubits from Alice to Bob,

$$
t_{N}=\frac{t_{0}}{N+1}=\frac{t_{0}}{4 k^{\prime}}, \quad k \gg 1 .
$$

We make some important remarks here. Once all AQS receive Alice's instruction messages, they begin executing their specific actions in parallel. For the overall distribution time, we do not account for the time it takes for AQS to receive their instructions. Given that entanglement swapping is the key principle of operation, the order in which it happens is not important because the entanglement propagates between nodes until it eventually reaches Alice and Bob. Therefore, Alice's particle from her EPR pair must only go through one hop, unlike standard distribution which requires $4 k$ hops.

The series of operations we have presented so far are related to the route from Alice to Bob. The protocol is symmetric, i.e., the analogous operations are performed from Bob's perspective. Hence, Alice and Bob are actually supplied with two ebits in the time interval $t_{N}$, one ebit from each route.

\section{EntangleNet Protocol}

Following the detailed description of its functionality in the previous section, we now present an algorithmic implementation of our protocol. The EntangleNet distribution scheme is presented in Algorithm 1 and it uses functions defined in Algorithms 2 and 3.

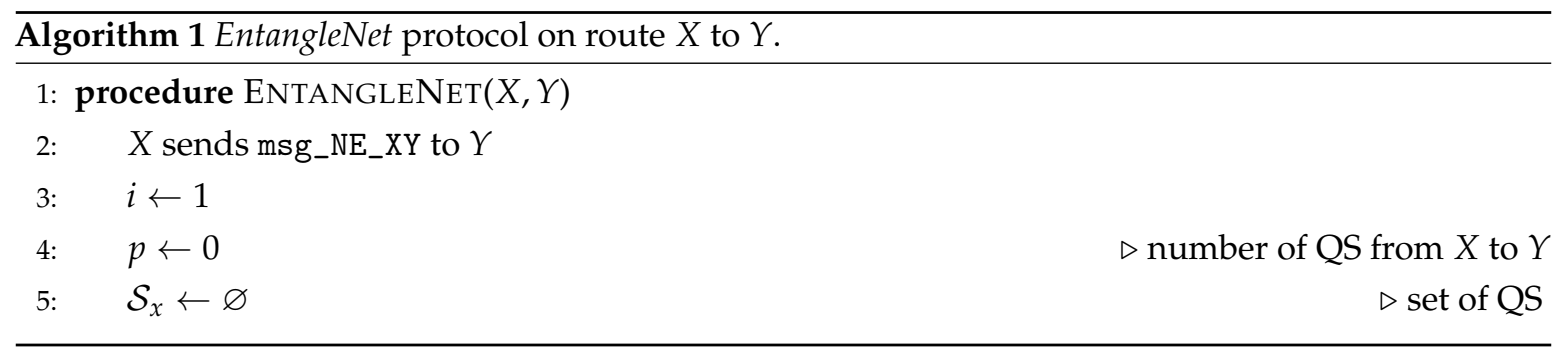




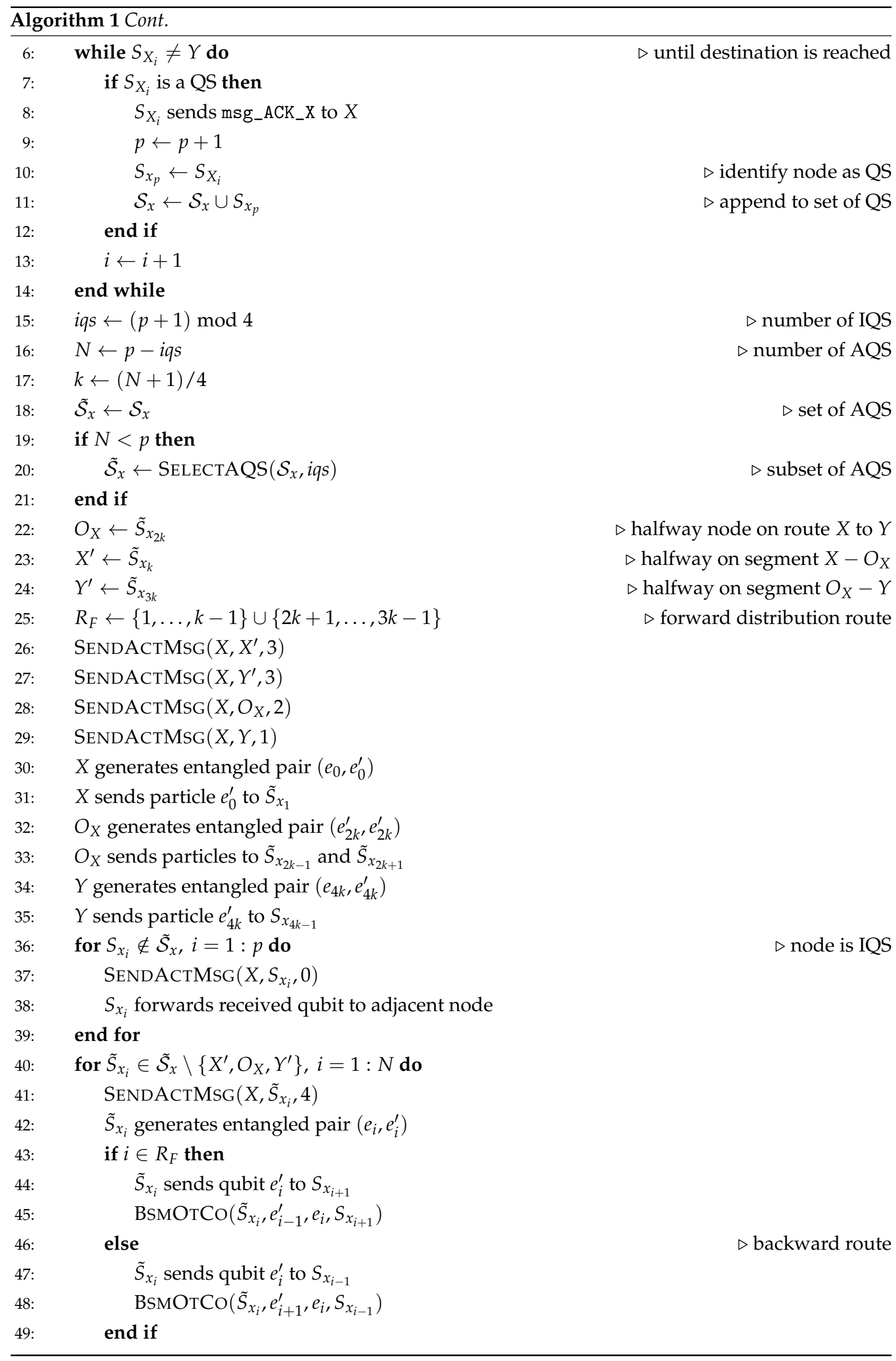



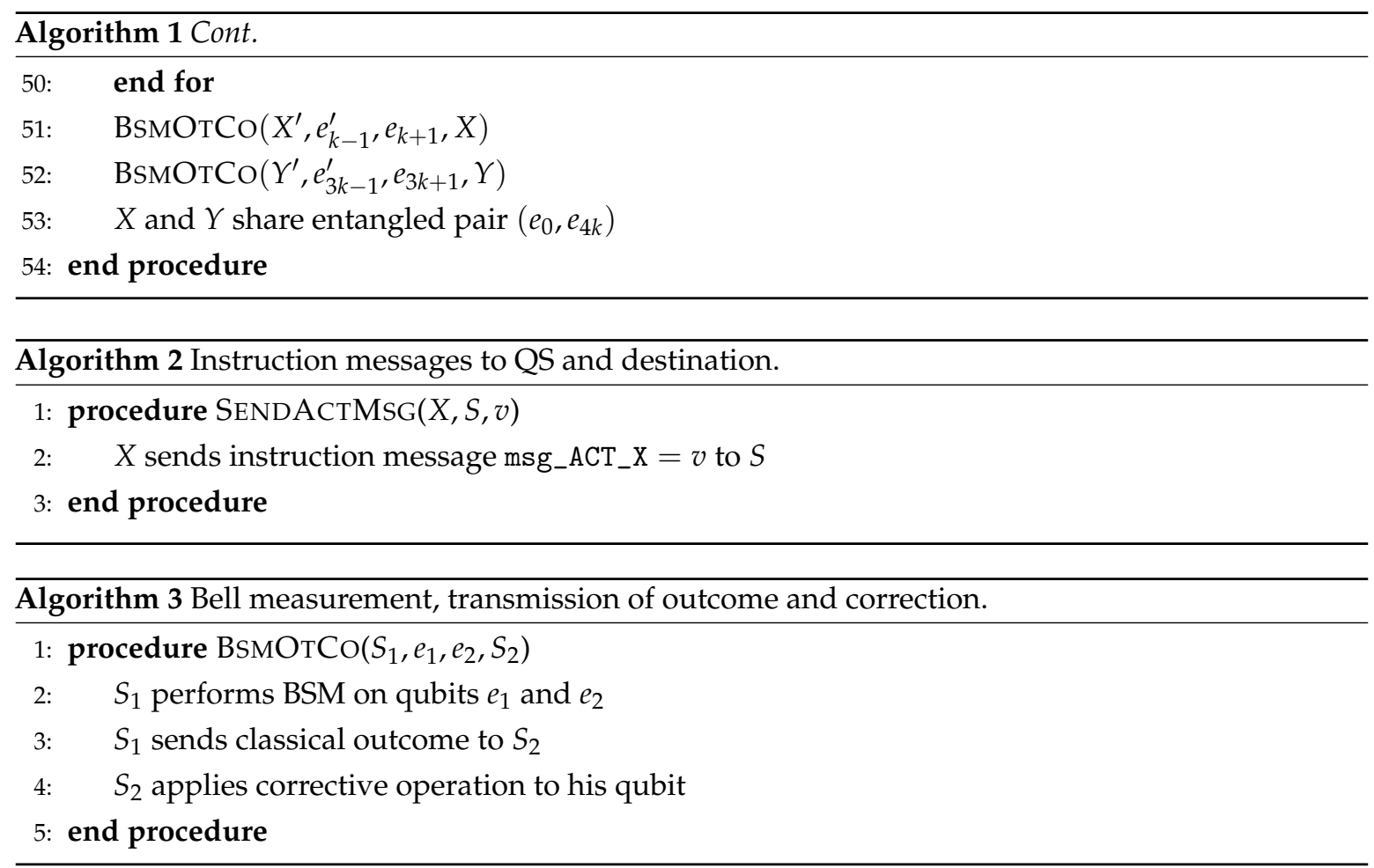

The main procedure of the protocol is described in a general manner that is applicable to any nodes $X$ and $Y$. Function SELECTAQS $\left(\mathcal{S}_{x}, i q s\right)$ is an abstract representation of the decision made by $X$ to extract a subset of quantum switches that will be actively involved in pair generation and measurements. The execution of the protocol for our parties Alice and Bob is given in Algorithm 4.

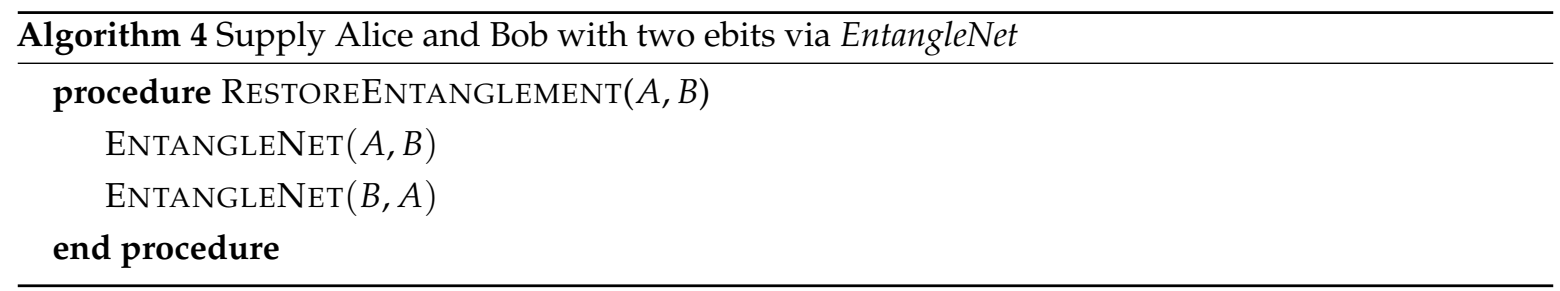

\section{Conclusions}

In this paper, we have presented a theoretical approach to a routing protocol that reestablishes the entangled resource between two arbitrary nodes $A$ and $B$ in a quantum network in order to be used further in teleportation. When compared to [9] and to the direct transmission of qubits from $A$ to $B$, our proposal benefits from a speedup in time given by a factor of $4 k$, increasing with the number of $N=4 k-1$ active quantum switches between the end nodes.

The protocol operates by transmission of classical instruction messages issued by Alice or Bob, distribution of entangled particles to adjacent nodes and specific operations executed at each node. The scheme takes advantage of nodes we refer to as active quantum switches, inactive quantum switches and classical switches. The first type employs entanglement swapping, while the other two mediate the flow of qubits by forwarding them to adjacent nodes, until an active quantum switch is reached. Entanglement swapping performed at each AQS eventually propagates entanglement towards Alice and Bob, who, after the completion of the protocol, get to share two ebits, one from each execution route, Alice to Bob and vice versa.

As a future development, we propose the physical implementation of this prototype of the protocol with our academic partners. 
Author Contributions: Conceptualization, M.-Z.M. and P.G.P.; Methodology, P.G.P.; Formal Analysis, P.G.P.; Investigation, M.-Z.M. and P.G.P.; Writing-Original Draft Preparation, M.-Z.M.; Writing-Review \& Editing, M.-Z.M. and P.G.P.; Supervision, P.G.P.

Funding: This research received no external funding

Conflicts of Interest: The author declares no conflict of interest.

\section{References}

1. Bennett, C.H.; Brassard, G.; Crépeau, C.; Jozsa, R.; Peres, A.; Wootters, W.K. Teleporting an unknown quantum state via dual classical and Einstein-Podolsky-Rosen channels. Phys. Rev. Lett. 1993, 70, 1895-1899. [CrossRef] [PubMed]

2. Bennett, C.H.; Bernstein, H.J.; Popescu, S.; Schumacher, B. Concentrating partial entanglement by local operations. Phys. Rev. A 1996, 53, 2046-2052. [CrossRef] [PubMed]

3. Lo, H.K.; Popescu, S. Classical Communication Cost of Entanglement Manipulation: Is Entanglement an Interconvertible Resource? Phys. Rev. Lett. 1999, 83, 1459-1462. [CrossRef]

4. Bennett, C.H.; DiVincenzo, D.P.; Smolin, J.A.; Wootters, W.K. Mixed-state entanglement and quantum error correction. Phys. Rev. A 1996, 54, 3824-3851. [CrossRef] [PubMed]

5. Popescu, S.; Rohrlich, D. Thermodynamics and the measure of entanglement. Phys. Rev. A 1997, 56, R3319-R3321. [CrossRef]

6. Vedral, V.; Plenio, M.B.; Rippin, M.A.; Knight, P.L. Quantifying Entanglement. Phys. Rev. Lett. 1997, 78, 2275-2279. [CrossRef]

7. Plenio, M.B.; Vedral, V. Teleportation, entanglement and thermodynamics in the quantum world. Contemp. Phys. 1998, 39, 431-446. [CrossRef]

8. Bennett, C.H.; Popescu, S.; Rohrlich, D.; Smolin, J.A.; Thapliyal, A.V. Exact and asymptotic measures of multipartite pure-state entanglement. Phys. Rev. A 2000, 63, 012307. [CrossRef]

9. Bose, S.; Vedral, V.; Knight, P.L. Multiparticle generalization of entanglement swapping. Phys. Rev. A 1998, 57, 822-829. [CrossRef]

10. Bennett, C.H.; Brassard, G.; Popescu, S.; Schumacher, B.; Smolin, J.A.; Wootters, W.K. Purification of Noisy Entanglement and Faithful Teleportation via Noisy Channels. Phys. Rev. Lett. 1996, 76, 722-725. [CrossRef] [PubMed]

11. Sheng, Y.B.; Zhou, L. Quantum Entanglement Concentration Based on Nonlinear Optics for Quantum Communications. Entropy 2013, 15, 1776-1820. [CrossRef]

12. Briegel, H.J.; Dür, W.; Cirac, J.I.; Zoller, P. Quantum Repeaters: The Role of Imperfect Local Operations in Quantum Communication. Phys. Rev. Lett. 1998, 81, 5932-5935. [CrossRef]

13. Yurke, B.; Stoler, D. Einstein-Podolsky-Rosen effects from independent particle sources. Phys. Rev. Lett. 1992, 68, 1251-1254. [CrossRef] [PubMed]

14. Żukowski, M.; Zeilinger, A.; Horne, M.A.; Ekert, A.K. “Event-ready-detectors” Bell experiment via entanglement swapping. Phys. Rev. Lett. 1993, 71, 4287-4290. [CrossRef] [PubMed]

15. Pan, J.W.; Bouwmeester, D.; Weinfurter, H.; Zeilinger, A. Experimental Entanglement Swapping: Entangling Photons That Never Interacted. Phys. Rev. Lett. 1998, 80, 3891-3894. [CrossRef]

16. Marshall, K.; Weedbrook, C. Continuous-Variable Entanglement Swapping. Entropy 2015, 17, 3152-3159. [CrossRef]

17. Zhang, Y.; Li, Z.; Weedbrook, C.; Marshall, K.; Pirandola, S.; Yu, S.; Guo, H. Noiseless Linear Amplifiers in Entanglement-Based Continuous-Variable Quantum Key Distribution. Entropy 2015, 17, 4547-4562. [CrossRef]

18. Zhang, Z.; Shi, R.; Guo, Y. Multipartite Continuous Variable Quantum Conferencing Network with Entanglement in the Middle. Appl. Sci. 2018, 8, 1312. [CrossRef] 
19. Caleffi, M. Optimal Routing for Quantum Networks. IEEE Access 2017, 5, 22299-22312. [CrossRef]

20. Cheng, S.T.; Wang, C.Y.; Tao, M.H. Quantum communication for wireless wide-area networks. IEEE J. Sel. Areas Commun. 2005, 23, 1424-1432. [CrossRef]

(C) 2018 by the authors. Licensee MDPI, Basel, Switzerland. This article is an open access article distributed under the terms and conditions of the Creative Commons Attribution (CC BY) license (http:/ / creativecommons.org/licenses/by/4.0/). 